\title{
INTENCIÓN DE EMPRENDIMIENTO EN LOS ESTUDIANTES: UN ESTUDIO EN LA UNIVERSIDAD CATÓLICA DE SANTIAGO DE GUAYAQUIL
}

\author{
INTENTION OF ENTREPRENEURSHIP IN STUDENTS: A STUDY AT THE \\ CATHOLIC UNIVERSITY OF SANTIAGO DE GUAYAQUIL
}

DANNY X. ARÉVALO AVECILLAS', CARMEN P. PADILLA LOZANO², NANCY I. WONG LABORDE³

\footnotetext{
Universidad Católica de Santiago de Guayaquil. danny.arevalo@cu.ucsg.edu.ec

Universidad Católica de Santiago de Guayaquil. carmen.padilla@cu.ucsg.edu.ec

3 Universidad Católica de Santiago de Guayaquil. nancy.wong@cu.ucsg.edu.ec
}

\begin{abstract}
A partir de datos tomados en el segundo semestre del año 2014 se comparan las actitudes personales, sociales y de entorno hacia el emprendimiento en la facultad de Ciencias Económicas y Administrativas (CEA). Se realiza una investigación no experimental, con horizonte de tiempo transversal descriptivo. Se tomó como base el modelo de intención de emprendimiento de Liñán, Urbano \& Guerrero (2011). Para el análisis estadístico se tomaron como base los datos de cinco carreras, donde la muestra ponderada fue de 328 estudiantes. Pese a que todas carreras tienen una predisposición por encima de la media, los estudiantes de Administración presentan mejores resultados y, a la vez, su carrera presenta mayor cantidad de inscritos con un $32 \%$ de la muestra. También se muestra cómo la universidad puede influir de forma determinante en la intención de emprendimiento. El estudio proporciona información valiosa para líderes de la administración privada, docentes, estudiantes y empresarios.
\end{abstract}

PALABRAS CLAVE: intención de emprendimiento, universidad, creación de empresas, Ciencias Económicas, Ciencias Administrativas.
ABSTRACT

From data collected in the second half of 2014 semester, social environment and attitudes toward entrepreneurship at the faculty of Economics and Administrative Sciences (CEA). A non-experimental research is carried out, with descriptive transversal time horizon. The entrepreneurial intention model of Linan, Urban \& Guerrero (2011) was taken as a basis. For statistical analysis on the data they took five careers, where the weighted sample was 328 students. Although all careers are predisposed above average, students of Administration have better results, while their career has registered the $32 \%$ of the sample. It also shows how the university can influence decisively in the intention of entrepreneurship. The study provides valuable information for private management leaders, teachers, students and businessmen.

KEYWORDS: entrepreneurship intention, university, entrepreneurship, Economics, Management Sciences. 
INTRODUCCIÓN

La importancia que el emprendimiento tiene en el desarrollo de una nación es latente y por ello es una necesidad en países en vías de desarrollo (Zoltan, 2006), razón por la cual, los gobiernos deben promover la educación en emprendimiento para expandir el alcance del mismo (Smit, 2004). Mediante el entrenamiento es posible crear emprendedores que ayuden a la expansión de la economía (Karimi, Chizari, Biemans, \& Mulder, 2010). Es importante que las políticas del estado tengan efecto en la formación universitaria para que las universidades ayuden a la creación de negocios de emprendimiento a través de sus programas educacionales y de investigación (Wallace \& Nilsson, 1997).

A continuación, se aborda la revisión de literatura acerca del emprendimiento en general y específicamente en el Ecuador, seguido del abordaje del modelo creado por Liñán, Urbano y Guerrero (2011) que se usa en la investigación junto con las conclusiones y recomendaciones para posteriores trabajos.

\section{EVOLUCIÓN DE LA PALABRA EMPRENDIMIENTO}

Como lo indica Rodríguez (2009) al describir la palabra "emprendimiento" se debe revisar el término francés entrepreneur, que significa estar listo a tomar decisiones o listo para iniciar algo. Dicho autor describe la evolución histórica del término basándose en Vérin (1982) en donde muestra cómo a partir de los siglos XVII y XVIII se calificaba de emprendedor al arquitecto y al maestro de obra. Según como lo define Rodríguez (2009):

La definición del término emprendedor ha ido evolucionando y se ha transformado con base en estas dos concepciones, hasta hoy en día, que caracteriza a la persona en un estado de innovación permanente, altamente motivada y comprometida con una tarea, que reporta unas características de planeación y ejecución, propensa al riesgo, y a la vez esquiva a la comprensión de sus propias dinámicas (p. 97).

Para Amit (1997), las investigaciones de percepciones de los ejecutivos describen el emprendimiento o su espíritu emprendedor con términos como ser creativo, innovador, capaz de asumir riesgos, flexible, dinámico, creativo, orientado al crecimiento y al cambio (Ajzen, 1991; Cruz, Barahona, y Escudero, 2005;
Delmar \& Davidsson, 2000; Olmos y Castillo, 2007; Reynolds, Hayand, \& Camp, 1999; Shapero \& Sokol, 1982). Algunos libros sobre administración han definido el término como la capacidad de formar y operar nuevas empresas (Bennis \& Nanus, 1985; Kantis, Angelelli, y Moori, 2004; Mintzberg, Ahlstrand, y Lampel, 1999).

\section{REPORTE DE GLOBAL ENTREPRENEURSHIP MONITOR 2014 (GEM - MONITOR GLOBAL DE EMPRENDIMIENTO)}

Este reporte proporciona los resultados del décimosexto ciclo de encuestas que se llevan a cabo cada año desde 1999.

Setenta y tres países participaron en esta encuesta en el 2014 cuyo informe proporciona los resultados en cuanto a atributos y actividades de setenta de estos países y sobre el ecosistema emprendedor de la totalidad (Laso, Caicedo, Ordeñana, \& Izquierdo, 2015).

Los países que participan en la encuesta Global Entrepreneurship Monitor, GEM 2014 representan el $72,4 \%$ de la población mundial y el $90 \%$ del PIB mundial, lo que proporciona una base muy importante para la identificación de las diferentes características del fenómeno del emprendimiento, tal como se expone en el marco conceptual utilizado (Laso et al., 2015).

El marco conceptual del GEM, tal como fue identificado en 1999, según lo muestra la Figura 1 , en contraste con el modelo convencional de crecimiento económico nacional que se muestra en la Figura 2, representa el supuesto básico de que este crecimiento es el resultado de la capacidad personal de los individuos para identificar y aprovechar las oportunidades (dondequiera que se encuentren y sin importar si trabajan por cuenta propia o del tamaño de las empresas), y que este proceso está tomando lugar en la interacción con el medio ambiente.

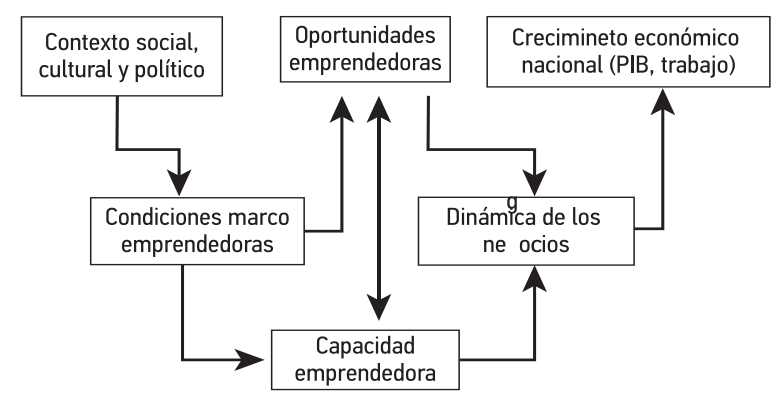

Figura 1. Modelo de los procesos emprendedores y su efecto en la economía.

Fuente: Singer, Amorós, \& Arreola (2015) 


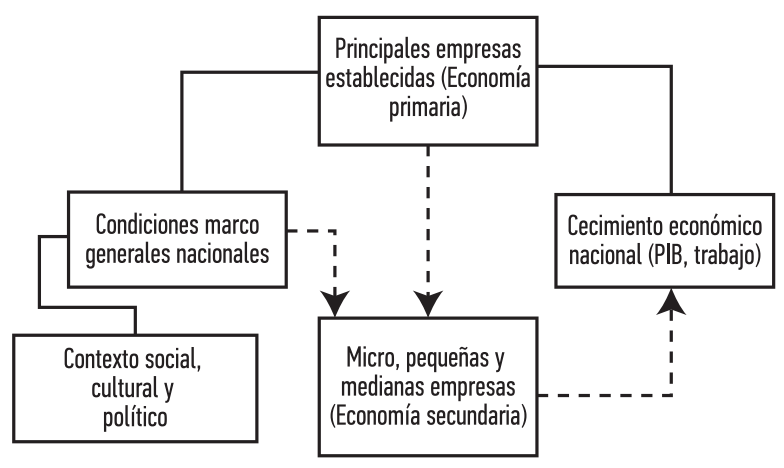

Figura 2. Modelo convencional de crecimiento económico nacional Fuente: Singer, Amorós, \& Arreola (2015)

Utilizando los resultados de las encuestas GEM a lo largo de los años, este marco conceptual inicial evolucionó al marco conceptual del CEM que se muestra en la Figura 3, tomada de Singer et al. (2015) que aborda el perfil del emprendedor.

\section{EMPRENDIMIENTO EN EL ECUADOR}

Según el Reporte Global Entrepreneurship Monitor, Informe Ecuador 2014, se indica que en el año 2014 se observó una reducción de la actividad emprendedora temprana con respecto al 2013, pasando la Tasa de Emprendimiento Anual (TEA) de $36 \%$ a $32.6 \%$ (Laso et al., 2015). En América Latina, Ecuador muestra nuevamente una de las TEA más alta, Tabla 1, seguido por Perú $(28.8 \%$ y Chile $(26.8 \%)$. Este valor también representa una de la TEA más alta entre las economías basadas en la eficiencia. Durante el año analizado, la TEA está compuesta por un $24.5 \%$ de negocios nacientes y un $9.9 \%$ de negocios nuevos.

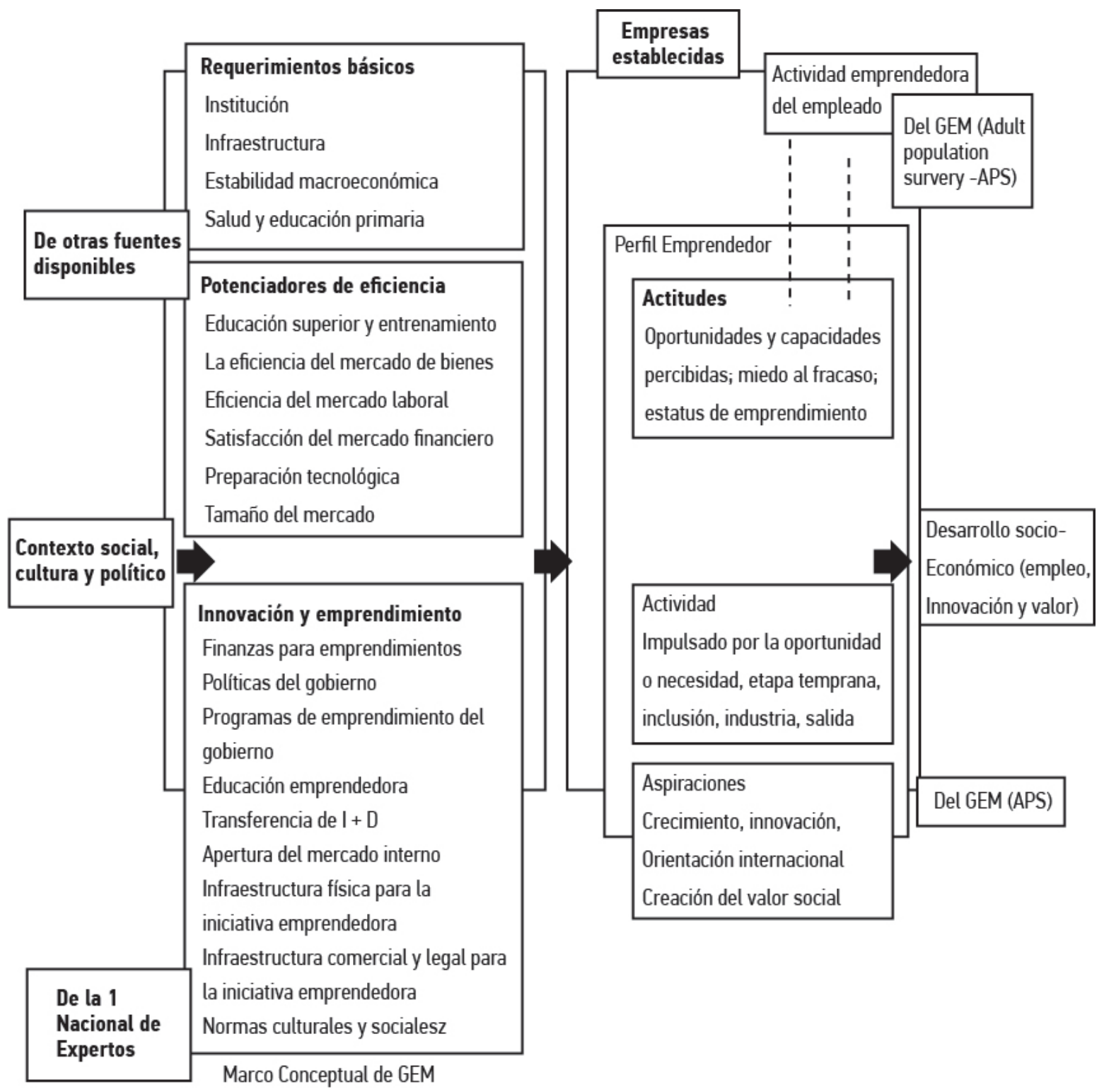

Figura 3. Marco Conceptual del Global Entrepreneurship Monitor, GEM. 


\section{TABLA 1. ACTIVIDAD EMPRENDEDORA Y PERCEPCIONES EN LAS ECONOMÍAS DEL GEM 2014, LATINOAMÉRICA Y CARIBE}

\begin{tabular}{|c|c|c|c|c|c|c|c|}
\hline PAÍs & $\begin{array}{l}\text { TASA DE } \\
\text { EMPRENDIMIENTOS } \\
\text { NACIENTES }\end{array}$ & $\begin{array}{l}\text { TASA DE } \\
\text { EMPRENDIMIENTOS } \\
\text { NUEVOS }\end{array}$ & $\begin{array}{l}\text { TASA DE } \\
\text { ACTIVIDAD } \\
\text { EMPRENDEDORA } \\
\text { TEMPRANA (TEA) }\end{array}$ & $\begin{array}{l}\text { TASA DE } \\
\text { NEGOCIOS } \\
\text { ESTABLECIDOS }\end{array}$ & $\begin{array}{l}\text { TASA DE CIERRE } \\
\text { DE NEGOC IOS }\end{array}$ & $\begin{array}{l}\text { TASA DE } \\
\text { EMPRENDIMIENTO } \\
\text { POR NECESIDAD } \\
\text { (\% DE LA TEA) }\end{array}$ & $\begin{array}{l}\text { TASA DE } \\
\text { EMPRENDIMIENTO } \\
\text { POR } \\
\text { OPORTUNIDAD DE } \\
\text { MEJORA (\% DE } \\
\text { LA TEA) }\end{array}$ \\
\hline Argentina & 9,5 & 5,2 & 14,4 & 9,1 & 4,9 & 28,0 & 43,5 \\
\hline Barbados & 8,5 & 4,2 & 12,7 & 7,1 & 3,7 & 14,6 & 53,1 \\
\hline Belice & 4,3 & 3,0 & 7,1 & 3,7 & 4,7 & 13,1 & 47,6 \\
\hline Bolivia & 21,5 & 7,1 & 27,4 & 7,6 & 6,9 & 22,8 & 51,7 \\
\hline Brasil & 3,7 & 13,8 & 17,2 & 17,5 & 4,1 & 29,0 & 57,8 \\
\hline Chile & 16,6 & 11,0 & 26,8 & 8,8 & 8,3 & 17,6 & 62,2 \\
\hline Colombia & 12,4 & 6,7 & 18,5 & 4,9 & 5,6 & 33,3 & 51,5 \\
\hline Costa Rica & 7,6 & 3,7 & 11,3 & 2,5 & 4,9 & 19,3 & 63,1 \\
\hline Ecuador & 24,5 & 9,9 & 32,6 & 17,7 & 8,1 & 29,4 & 34,9 \\
\hline El Salvador & 11,4 & 8,7 & 19,5 & 12,7 & 10,8 & 31,9 & 54,5 \\
\hline Guatemala & 12,0 & 9,2 & 20,4 & 7,4 & 4,4 & 40,6 & 38,9 \\
\hline Jamaica & 7,9 & 11,9 & 19,3 & 14,4 & 6,3 & 32,1 & 33,5 \\
\hline México & 12,7 & 6,4 & 19,0 & 4,5 & 5,6 & 22,5 & 50,0 \\
\hline Panamá & 13,1 & 4,1 & 17,1 & 3,4 & 4,5 & 26,3 & 60,2 \\
\hline Perú & 23,1 & 7,3 & 28,8 & 9,2 & 8,0 & 16,4 & 58,9 \\
\hline Puerto Rico & 8,8 & 1,3 & 10,0 & 1,3 & 3,6 & 20,5 & 51,1 \\
\hline Surinam & 1,9 & 0,2 & 2,1 & 5,2 & 0,2 & 5,4 & 39,8 \\
\hline $\begin{array}{l}\text { Trinidad \& } \\
\text { Tobago }\end{array}$ & 7,5 & 7,4 & 14,6 & 8,5 & 2,8 & 12,0 & 64,3 \\
\hline Uruguay & 10,5 & 5,7 & 16,1 & 6,7 & 4,4 & 16,0 & 27,3 \\
\hline $\begin{array}{l}\text { Promedio } \\
\text { (ponderado) }\end{array}$ & 11,4 & 6,7 & 17,6 & 8,0 & 5,4 & 22,7 & 49,7 \\
\hline
\end{tabular}

Fuente: Laso et al. (2015).

Vale la pena destacar que la actividad emprendedora naciente corresponde a individuos iniciando una empresa en menos de tres meses de antigüedad. Dados los desafíos asociados con el inicio de un nuevo negocio, muchos nacientes fracasan en los primeros meses. Por lo tanto, no todos los emprendedores avanzan a la siguiente etapa.

Los nuevos emprendedores se definen como aquellos antiguos emprendedores nacientes que han estado en el negocio durante más de tres meses, pero menos de tres años y medio o 42 meses (Laso et al., 2015). Un $22.8 \%$ de los emprendedores está motivado por la oportunidad, similar a los resultados obtenidos en el año 2013, pero la TEA por necesidad se ha reducido a $9.6 \%$, nivel comparable al obtenido en 2012. Ver Figura 4 tomada de Laso et al. (2015).

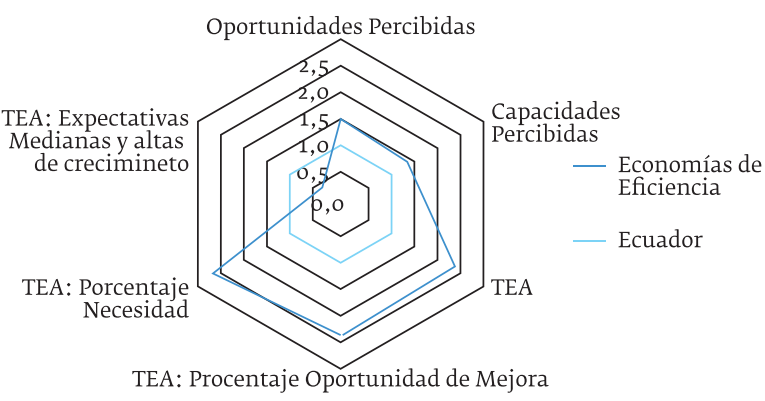

Figura 4. Perfil del emprendimiento para Ecuador y el promedio de las economías de eficiencia

A pesar de que Ecuador tiene la segunda TEA por oportunidad más alta de la región, después de Perú, la TEA por oportunidad representa un $70 \%$ de la TEA total, encontrándose por debajo de la media de América Latina y el Caribe. Por el contrario, en 2014, existe una alta proporción de negocios iniciados por necesidad, duplicando aproximadamente las de Perú y Chile, Figuras 5 y 6 . 


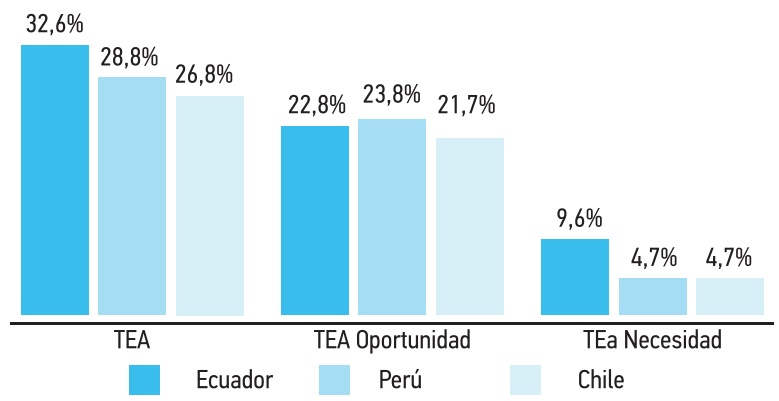

Figura 5. Contraste de TEA por países según motivación. Fuente: Laso et al. (2015).

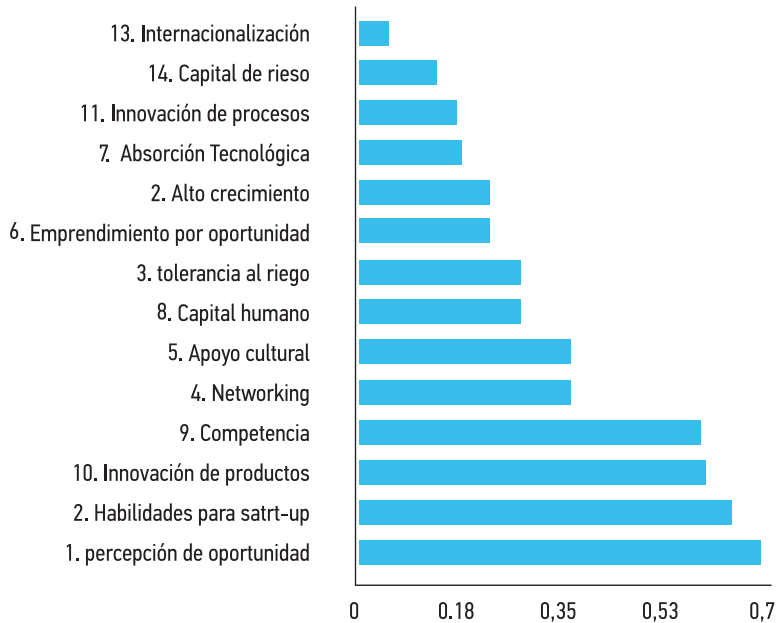

Figura 6. Pilares del Global Entrepreneurship Index (GEI) para Ecuador 2015 , en orden ascendente.

Fuente: Laso et al. (2015).

En base a lo encontrado sobre los pilares del emprendimiento en el Ecuador, Figura 6, el informe Global Entrepreneurship Monitor Ecuador 2014 (Laso et al., 2015) indica que las políticas claves a seguir para mejorar las condiciones del emprendimiento serían aquellas orientadas a:

a. Fomento: de la internacionalización del emprendimiento a través de los organismos como Proecuador, Ministerio de Comercio Exterior y las embajadas.

b. Financiamiento: se requieren establecer políticas que estén orientadas a las distintas etapas del emprendimiento, capital semilla, capital de riesgo, inversiones ángeles y el fomento de un mercado bursátil (ya desarrollado por los vecinos Perú y Colombia).

c. Innovación tecnológica: como fue comentado anteriormente, los incentivos a I+D en las empresas establecidas pueden ayudar a incrementar la innovación. Dar los espacios para que a través del ecosistema de emprendimiento se pueda dar un efecto spill-over de la innovación. También, el acceso a nuevos mercados - a través de acuerdo de comercio- obliga a las empresas nacionales, tanto nuevas como establecidas, a aumentar sus esfuerzos en innovación, a fin de ser capaces de competir con empresas internacionales.

Las tres políticas anteriores confluyen en una política orientada hacia conseguir dinamismo o alto crecimiento en los emprendimientos. Empresas más innovadoras, con capacidad de capitalización y orientadas hacia un mercado exterior necesitarán poco más para crecer y mantenerse en el tiempo (p. 59).

\section{EL MODELO DE INTENCIÓN DE EMPRENDIMIENTO}

La investigación en emprendimiento, según Liñán y otros (2011), se basa en dos líneas importantes: (a) las características del individuo y (b) la influencia de su entorno. De esta manera la gestión de la información obtenida debe ser adecuada a lo que se busca generar. Al emplear la técnica de estos autores es importante tomar en cuenta el cuestionario usado en su trabajo. Con la intención de compartir una interpretación del modelo, Montiel, Márquez, Arámbula y Ordóñez (2012) aclaran que:

Mitchell, Busenitz, Lant, Mcdougall, Morse \& Smith (2002) mencionan que los principales modelos con una base cognitiva que han servido para explicar el emprendimiento son el del evento empresarial (Shapero \& Sokol, 1982) y el de la teoría de la conducta planeada (Ajzen, 1991). El primero se enfoca en el evento empresarial, condicionado este por las percepciones de lo atractivo (el sistema individual de valores y el sistema social del cual el individuo es parte) y factibilidad (financiamiento y socios potenciales). Percepciones producto de ambientes sociales y culturales, los cuales determinan las elecciones personales (Shapero \& Sokol, 1982). Por su parte, el modelo de (Ajzen, 1991) aborda como el medio ambiente cultural y social afecta la conducta, normas subjetivas y el control percibido de comportamiento (Perceived Behavioural Control, por sus siglas en inglés, $\mathrm{PBC}$ ). En base a estos dos modelos y a la falta de evidencia sobre variaciones entre regiones o países (Delmar \& Davidsson, 2000), Liñán et al. (2011), desarrollaron un modelo de intención de emprendimiento, integrado por tres factores: motivacionales, ambientales y situacionales (variables de control). (p. 1233).

A continuación, se presenta el modelo desarrollado por Liñán et al. (2011) junto con las 
hipótesis que serán testeadas en el presente estudio, Figura 7:

- Hla: la actitud hacia la conducta tendrá un impacto positivo en la intención de emprendimiento, independientemente del contexto regional.

- H1b: el control percibido de conducta tendrá un impacto positivo en la intención de emprendimiento independientemente del contexto regional.

- H2a: las normas subjetivas tendrán un impacto positivo en la actitud hacia la conducta independientemente de su contexto regional.

- H2b: las normas subjetivas tendrán un impacto positivo en el control percibido de la conducta independientemente del contexto regional.

- H3a: la valoración cercana tendrá un impacto positivo en la actitud hacia la conducta, pero es diferente dependiendo del contexto regional.

- H3b: la valoración cercana tendrá un impacto positivo en las normas subjetivas, pero es diferente dependiendo del contexto regional.

- H3c: la valoración social tendrá un impacto positivo en las normas subjetivas, pero es diferente dependiendo del contexto regional.

- H3d: la valoración social tendrá un impacto positivo en el control percibido de la conducta, pero es diferente dependiendo del contexto regional

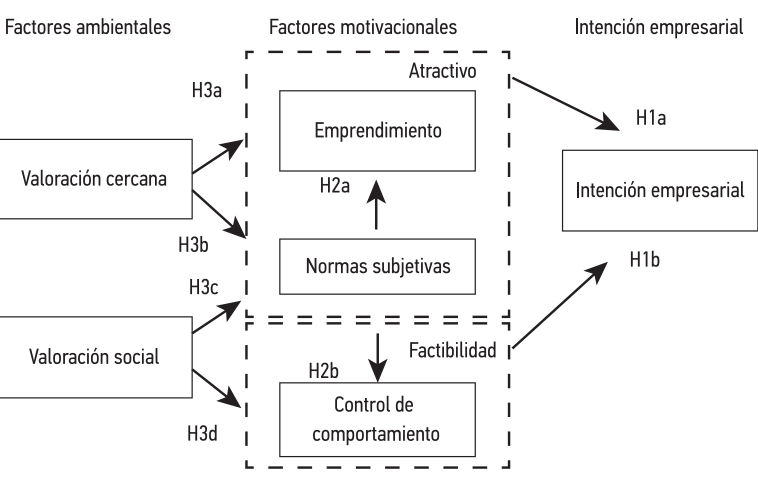

Figura 7: Modelo de emprendimiento

METODOLOGÍA

La presente investigación es no experimental, con horizonte de tiempo transversal de tipo descriptiva. La estrategia de la investigación es el estudio de caso (Galicia, 1990; Montiel y otros, 2012; Hernández, Fernández, y Baptista, 2010; Samaja, 2007). "Según Yin (2003) esta estrategia se puede usar para un enfoque cualitativo o cuantitativo, incluso mixto, para responder al tipo de preguntas de cómo y por qué» (Montiel y otros, 2012, p. 1235); en donde el estudio se desarrolla sobre un solo proceso o acontecimiento, con una unidad de análisis.

A continuación, se presenta el estudio de caso con los cinco pasos que Yin (2003) recomienda "pregunta de investigación, proposiciones, unidad de análisis, lógica para empatar datos a las proposiciones y el criterio para interpretar los hallazgos" (Montiel y otros, 2012, p. 1235). Describiendo así, nuestro problema de investigación: ¿Cómo se diferencia la intención de emprendimiento en los alumnos de las diferentes carreras de la facultad de Ciencias Económicas y Administrativas (CEA) de la Universidad Católica de Santiago de Guayaquil (UCSC)? Se busca identificar y explicar el entorno y los incentivos personales que puedan explicar las diferencias en la intención de emprendimiento en las $\mathrm{ca}^{-}$ rreras de la CEA, en base al modelo propuesto por Liñán et al. (2011). Los datos se recolectan en el semestre B-2014 en la UCSC. Acerca de los dos últimos pasos planteados por Yin, que hablan de la lógica para empatar datos a las proposiciones y el criterio para interpretar los hallazgos se ha empleado el punto de vista de los autores.

La muestra "que es una porción o parte de la población de interés" (Lind, 2012, p. 266), se presenta de manera ponderada en la Tabla 2, dadas las cantidades diferentes en las inscripciones para el semestre.

tABLA 2. MUESTRA PONDERAdA DE LOS ENCUESTAdos PARA INVESTIGACIÓN EN BASE A ALUMNOS MATRICULADOS EN EL SEMESTRE B-2014

\begin{tabular}{lccc}
\hline CARRERA & INSCRITOS & PORCENTAJE & $\begin{array}{l}\text { MUESTRA } \\
\text { PONDERADA }\end{array}$ \\
\hline $\begin{array}{l}\text { Ingeniería en } \\
\text { Empresas }\end{array}$ & 65 & $3 \%$ & 10 \\
$\begin{array}{l}\text { Administración } \\
\text { de Empresas }\end{array}$ & 686 & $32 \%$ & 103 \\
\hline CPA & 517 & $24 \%$ & 78 \\
Economía & 277 & $13 \%$ & 42 \\
$\begin{array}{l}\text { Gestión } \\
\text { Empresarial }\end{array}$ & 630 & $29 \%$ & 95 \\
Total & & & 328 \\
\hline
\end{tabular}

Liñán et al. (2011) agregan que los ítems A1-A20 miden los cuatro constructos centrales de la teoría de la conducta planteada plateada por Ajzen (1991), además, se toman en cuenta los valores sociales sobre emprendimiento con 8 ítems (C1-C8). En la Tabla 3 se presentan los ítems que nutren cada constructo estudiado. 
TABLA 3. CONSTRUCTOS EMPRESARIALES Y SOCIALES USADOS EN EL ESTUDIO

\begin{tabular}{ll}
\hline CONSTRUCTOS & ÍTEMS EN LA ENCUESTA \\
\hline $\begin{array}{l}\text { Intención empresarial } \\
\text { (A4, A6, A9-reversed (rev)-, A13, A17 } \\
\text { y A19-rev-) } \\
\text { Actitud hacia la conducta }\end{array}$ & (A2-rev-, A10, A12-rev-, A15 y A18) \\
\hline $\begin{array}{l}\text { Control percibido de la conducta } \\
\text { (PBC) }\end{array}$ & (A1, A5-rev-, A7, A14, A16-rev-, A20) \\
\hline $\begin{array}{l}\text { Normas subjetivas } \\
\text { Valoración del emprendimiento en } \\
\text { el ambiente }\end{array}$ & $\begin{array}{l}\text { (A3, A8 y A11) } \\
\text { valoración cercana) }\end{array}$ \\
\hline $\begin{array}{l}\text { Percepciones acerca de la } \\
\text { valoración social generalizada del } \\
\text { emprendimiento }\end{array}$ & (C2, C3-rev-, C5-rev-, C6, C8-rev-) \\
\hline
\end{tabular}

Fuente: Méndez, Miramontes, \& Monreal (2012).

RESULTADOS

La CEA cuenta con cinco carreras de margen económico y administrativo. En la Tabla 4 se presentan las características de la muestra obtenida para el presente trabajo.

TABLA 4. CARACTERÍSTICAS DE LA MUESTRA A PARTIR DE LA ENCUESTA REALIZADA A ALUMNOS MATRICULADOS EN EL SEMESTRE B-2014 DE LA UCSG

\begin{tabular}{lrrrrrrrr}
\hline & 19,00 & 20,20 & 20,29 & 20,50 & 20,36 & 20,07 \\
\hline $\begin{array}{l}\text { CARRERA } \\
\text { Edad }\end{array}$ & 1,67 & 1,58 & 1,66 & 1,64 & 1,65 & 1,64 \\
\hline $\begin{array}{l}\text { Sexo } \\
\text { ¿Cuál es el nivel de } \\
\text { estudios alcanzado } \\
\text { por su padre? }\end{array}$ & 1,92 & 2,41 & 2,54 & 2,43 & 2,36 & 2,33 \\
$\begin{array}{l}\text { ¿Cuál es el nivel de } \\
\text { estudios alcanzado } \\
\text { por su madre? }\end{array}$ & 2,50 & 2,26 & 2,52 & 2,48 & 2,40 & 2,43 \\
$\begin{array}{l}\text { ¿Cuál es la ocupación } \\
\text { actual de su padre? }\end{array}$ & 2,00 & 2,19 & 2,06 & 2,32 & 2,18 & 2,15 \\
$\begin{array}{l}\text { Cuál es la ocupación } \\
\text { actual de su madre? }\end{array}$ & 2,67 & 3,00 & 2,83 & 2,89 & 2,73 & 2,82 \\
\hline $\begin{array}{l}\text { Número de personas } \\
\text { que residen en su } \\
\text { domicilio (incluyendo } \\
\text { a usted) }\end{array}$ & 5,25 & 4,92 & 5,29 & 5,09 & 5,00 & 5,11 \\
\hline
\end{tabular}

En cuanto a las características descriptivas de la muestra, el promedio de edad es de 20,07 y se encuestó mayormente a mujeres (1=hombre, $2=$ mujer). En el hogar de los estudiantes, sus madres presentan una formación más cercana al tercer nivel de educación que los padres ( $2=$ secundaria, $3=$ =universitaria). Los trabajos de los padres se concentran en el sector público, en cambio, las madres tienen una actitud emprendedora (2=servidor público, $3=$ emprendimiento). El rango de ingresos se encuentra entre el salario mínimo vital período 2014 (\$340) y los \$2000 mensuales. Los alumnos entrevistados fueron, en su mayoría, nuevos elementos en el sistema educativo universitario, puesto que el promedio semestral de la muestra es 2,28 que significa que la mayoría está entre el segundo y tercer semestre de estudios.

En cuanto a la intención empresarial (desde 1 desacuerdo total a 7, acuerdo total), la Tabla 5 presenta información que arroja los elementos donde las carreras de Administración de Empresas y Economía, presentan mejores resultados de forma general. Los estudiantes de Economía, con un resultado que sobrepasa la media, toman la delantera en la disposición hacia el emprendimiento y la decisión para crear una empresa, mientras que tienen mayor seguridad para decidir iniciar un negocio. Administración de Empresas presenta mayor predisposición para la creación y dirección de una empresa propia y tiene menos dudas con relación a su creación. Ingeniería en Empresas, por su parte, lidera en el ámbito de tener como objetivo ser emprendedor.

TABLA 5. INTENCIÓN EMPRESARIAL A PARTIR DE LA ENCUESTA REALIZADA A ALUMNOS MATRICULADOS EN EL SEMESTRE B-2014 DE LA UCSG

\begin{tabular}{|c|c|c|c|c|c|c|}
\hline CARRERA & 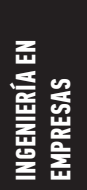 & 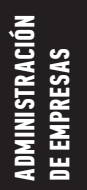 & 동 & 言 & 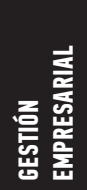 & 覓 \\
\hline $\begin{array}{l}\text { Estoy dispuesto a } \\
\text { hacer cualquier cosa } \\
\text { para ser emprendedor }\end{array}$ & 3,17 & 3,46 & 3,46 & 3,59 & 3,58 & 3,45 \\
\hline $\begin{array}{l}\text { Voy a hacer todo lo } \\
\text { posible para crear } \\
\text { y dirigir mi propio } \\
\text { negocio }\end{array}$ & 4,33 & 4,39 & 4,18 & 4,16 & 4,31 & 4,27 \\
\hline $\begin{array}{l}\text { Tengo algunas dudas } \\
\text { acerca de iniciar mi } \\
\text { propio negocio }\end{array}$ & 2,75 & 2,53 & 2,55 & 2,55 & 2,58 & 2,59 \\
\hline $\begin{array}{l}\text { Estoy decidido a crear } \\
\text { una empresa nueva en } \\
\text { el futuro }\end{array}$ & 3,67 & 4,34 & 3,82 & 4,59 & 4,45 & 4,17 \\
\hline $\begin{array}{l}\text { Mi objetivo profesional } \\
\text { es ser un emprendedor }\end{array}$ & 4,50 & 4,14 & 3,97 & 4,41 & 3,88 & 4,18 \\
\hline $\begin{array}{l}\text { Tengo una baja } \\
\text { intención de iniciar un } \\
\text { negocio }\end{array}$ & 2,17 & 1,88 & 1,82 & 1,73 & 2,01 & 1,92 \\
\hline $\begin{array}{l}\text { Número de personas } \\
\text { que residen en su } \\
\text { domicilio (incluyendo } \\
\text { a usted) }\end{array}$ & 5,25 & 4,92 & 5,29 & 5,09 & 5,00 & 5,11 \\
\hline
\end{tabular}


Con relación a la actitud hacia la conducta (desde 1 desacuerdo total a 7, acuerdo total), la carrera de Administración de Empresas presenta los datos más favorables como se presenta en la Tabla 6. En resumen, en todas las carreras se sugiere la gran satisfacción que generaría en el alumnado el hecho de ser emprendedor. La idea de tener más ventajas que desventajas se sitúa un poco más arriba que la neutralidad.

\section{TABLA 6. ACTITUD HACIA LA CONDUCTA A PARTIR DE LA ENCUESTA REALIZADA A ALUMNOS MATRICULADOS EN EL SEMESTRE B-2014 DE LA UCSG}

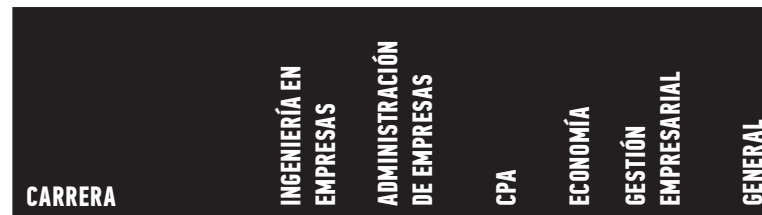

Una carrera como

empresario/a es

totalmente poco

atractivo para mí

$2,00 \quad 2,47 \quad 2,43 \quad 2,66 \quad 2,29 \quad 2,37$

\begin{tabular}{|c|c|c|c|c|c|c|}
\hline $\begin{array}{l}\text { Si tuviera la oportu- } \\
\text { nidad y recursos, me } \\
\text { gustaría iniciar un } \\
\text { negocio }\end{array}$ & 4,42 & 5,27 & 5,2 & 5,07 & 5,18 & 5,03 \\
\hline
\end{tabular}

Entre todas las

opciones, yo preferiría

$\begin{array}{lllllll}\text { ser cualquier cosa } & 2,25 & 1,95 & 1,91 & 1,75 & 2,01 & 1,97\end{array}$

menos empresario

\begin{tabular}{|c|c|c|c|c|c|c|}
\hline $\begin{array}{l}\text { Ser emprendedor } \\
\text { me daría una gran } \\
\text { satisfacción }\end{array}$ & 5,75 & 5,70 & 5,78 & 5,52 & 5,76 & 5,70 \\
\hline
\end{tabular}

Ser emprendedor

significa para mi tener

$\begin{array}{lllllll}\text { más ventajas que } & 3,50 & 4,28 & 4,22 & 3,80 & 3,85 & 3,93\end{array}$

desventajas

\begin{tabular}{|c|c|c|c|c|c|c|}
\hline $\begin{array}{l}\text { intención de iniciar un } \\
\text { negocio }\end{array}$ & 2,17 & 1,88 & 1,82 & 1,73 & 2,01 & 1,92 \\
\hline
\end{tabular}

\section{Número de personas}

que residen en su

domicilio (incluyendo $\quad 5,25 \quad 4,92 \quad 5,29 \quad 5,09 \quad 5,00 \quad 5,11$

a usted)

El PBC (desde 1 desacuerdo total a 7, acuerdo total) se presenta en la Tabla 7. Los resultados generales proyectan capacidades para iniciar un nuevo negocio. Adicionalmente, se presenta una alta aversión al riesgo de crear un negocio y, asimismo, la decisión de crearlo depende mucho de la probabilidad de su éxito.
TABLA 7. CONTROL PERCIBIDO DE LA CONDUCTA (PBC) A PARTIR DE LA ENCUESTA REALIZADA A ALUMNOS MATRICULADOS EN EL SEMESTRE B-2014 DE LA UCSG

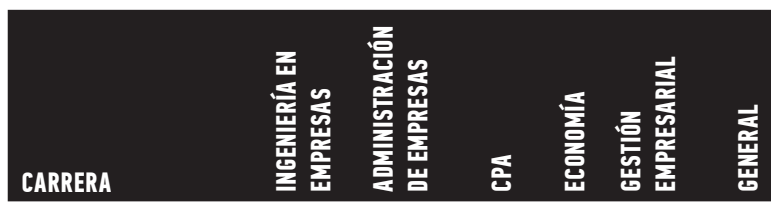

Abrir una empresa y $\begin{array}{lllllll}\text { mantenerla a largo } & 4,50 & 4,59 & 4,60 & 4,41 & 4,81 & 4,58\end{array}$

mí

Creo que yo sería

totalmente incapaz de $\quad 2,58 \quad 3,56 \quad 3,55 \quad 3,2 \quad 2,88 \quad 3,16$

iniciar un negocio

Soy capaz de controlar

$\begin{array}{llllll}\text { el proceso de creación } \quad 4,75 & 5,01 & 4,49 & 5,23 & 4,59 & 4,81\end{array}$

de un nuevo negocio

Si tratase de iniciar un

negocio, me gustaría

$\begin{array}{llllll}\text { tener mucha probabili- } \quad 6,42 & 5,72 & 6,09 & 6,02 & 5,57 & 5,96\end{array}$

dad de ser exitoso

Sería muy difícil para

mí desarrollar una idea $\quad 5,58 \quad 5,59 \quad 5,72 \quad 5,73 \quad 5,53 \quad 5,63$

de negocios

Sé todos los detalles

prácticos para iniciar $\quad 3,83 \quad 3,58 \quad 3,54 \quad 2,61 \quad 3,46 \quad 3,40$

un negocio

Número de personas

que residen en su

domicilio (incluyendo

$5,25 \quad 4,92 \quad 5,29 \quad 5,09 \quad 5,00 \quad 5,11$

a usted)

La Tabla 8 muestra las normas subjetivas (desde 1 desacuerdo total a 7, acuerdo total) y, como la familia es el principal pilar para la actitud emprendedora, seguido de los amigos y por último, los compañeros. Siendo altos, los tres valores sociales o puntajes.

TABLA 8. NORMAS SUBJETIVAS A PARTIR DE LA ENCUESTA REALIZADA A ALUMNOS MATRICULADOS EN EL SEMESTRE B-2014 DE LA UCSG

\begin{tabular}{|c|c|c|c|c|c|c|}
\hline CARRERA & 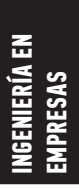 & 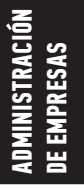 & 중 & 吾 & 它兽 & 惫 \\
\hline $\begin{array}{l}\text { Mis amigos aprobarían } \\
\text { mi decisión de iniciar } \\
\text { un negocio }\end{array}$ & 6,50 & 5,76 & 5,83 & 6,07 & 5,76 & 5,98 \\
\hline $\begin{array}{l}\text { Mi familia aprobaría mi } \\
\text { decisión de iniciar un } \\
\text { negocio }\end{array}$ & 6,50 & 6,50 & 6,68 & 6,32 & 6,62 & 6,52 \\
\hline $\begin{array}{l}\text { Mis compañeros } \\
\text { aprobarían mi decisión } \\
\text { de iniciar un negocio }\end{array}$ & 5,92 & 5,41 & 5,35 & 5,64 & 5,67 & 5,60 \\
\hline
\end{tabular}


En cuanto a la valoración social (desde 1 desacuerdo total a 7, acuerdo total), la Tabla 9 muestra que los compañeros apoyan la actitud emprendedora por encima de amigos y familia, aún así, los resultados de éstos son buenos para medir el apoyo que reciben los encuestados. Según los encuestados, en Ecuador la cultura emprendedora es aceptada $y$, agregan que, la sociedad considera que ser empresario es sinónimo de aprovecharse de los demás.

TABLA 9. VALORES SOCIALES SOBRE EMPRENDIMIENTO (VALUACIÓN CERCANA Y VALUACIÓN SOCIAL GENERAL) A PARTIR DE LA ENCUESTA REALIZADA A ALUMNOS MATRICULADOS EN EL SEMESTRE B-2014 DE LA UCSG

\begin{tabular}{|c|c|c|c|c|c|c|}
\hline CARRERA & 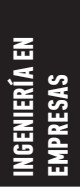 & 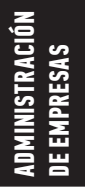 & 동 & 重 & 豆兽 & 業 \\
\hline $\begin{array}{l}\text { Mi familia valora la } \\
\text { actividad empresarial } \\
\text { por encima de otras } \\
\text { actividades y carreras }\end{array}$ & 4,33 & 4,54 & 4,05 & 4,59 & 4,14 & 4,33 \\
\hline $\begin{array}{l}\text { La cultura de mi país } \\
\text { es muy favorable hacia } \\
\text { la actividad empresarial }\end{array}$ & 3,25 & 4,78 & 4,2 & 4,57 & 4,44 & 4,25 \\
\hline $\begin{array}{l}\text { El papel del empresario } \\
\text { en la economía general } \\
\text { de mi país es subes- } \\
\text { timado }\end{array}$ & 2,00 & 2,85 & 2,65 & 3,09 & 2,70 & 2,66 \\
\hline $\begin{array}{l}\text { Mis amigos valoran la } \\
\text { actividad empresarial } \\
\text { por encima de otras } \\
\text { actividades y carreras }\end{array}$ & 5,83 & 5,45 & 5,26 & 5,50 & 5,38 & 5,49 \\
\hline $\begin{array}{l}\text { La mayoría de la gente } \\
\text { en mi país considera } \\
\text { inaceptable ser un } \\
\text { empresario }\end{array}$ & 3,25 & 2,35 & 2,02 & 2,41 & 1,95 & 2,39 \\
\hline $\begin{array}{l}\text { En mi país, se } \\
\text { considera que la } \\
\text { actividad empresarial } \\
\text { vale la pena a pesar de } \\
\text { los riesgos }\end{array}$ & 3,92 & 4,50 & 4,57 & 4,25 & 3,87 & 4,22 \\
\hline $\begin{array}{l}\text { Mis compañeros } \\
\text { valoran la actividad } \\
\text { empresarial por encima } \\
\text { de otras actividades y } \\
\text { carreras }\end{array}$ & 6,08 & 6,04 & 6,09 & 5,84 & 6,27 & 6,07 \\
\hline $\begin{array}{l}\text { Se piensa comúnmente } \\
\text { en mi país que los } \\
\text { empresarios se } \\
\text { aprovechan de los } \\
\text { demás }\end{array}$ & 5,42 & 5,71 & 5,31 & 5,23 & 5,56 & 5,44 \\
\hline
\end{tabular}

En el grado de atracción (desde 1 desacuerdo total a 7, acuerdo total) en la Tabla 10, la opción de ser empresario presenta datos significativos por encima de la opción de ser empleado. La predisposición hacia la creatividad, desarrollo, relaciones y liderazgo es mediana. Hay una tendencia mayor en la posibilidad de convertirse en empresario (1- si, 2- no, su media seria 1.5).

\section{TABLA 10. GRADO DE ATRACCIÓN Y CAPACIDADES ESPECÍFICAS A PARTIR DE LA ENCUESTA REALIZADA A ALUMNOS MATRICULADOS EN EL SEMESTRE B-2014 DE LA UCSG}

\begin{tabular}{|c|c|c|c|c|c|c|}
\hline CARRERA & 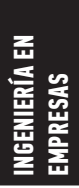 & 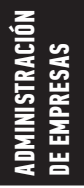 & 동 & 重 & 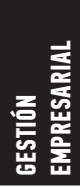 & 总 \\
\hline $\begin{array}{l}\text { Grado de atracción para } \\
\text { ser empleado }\end{array}$ & 2,33 & 2,43 & 2,32 & 2,59 & 2,86 & 2,51 \\
\hline $\begin{array}{l}\text { Grado de atracción por } \\
\text { ser empresario }\end{array}$ & 6,00 & 5,50 & 5,52 & 5,45 & 5,68 & 5,63 \\
\hline $\begin{array}{l}\text { Nivel de detección de } \\
\text { oportunidades }\end{array}$ & 4,83 & 4,90 & 4,91 & 5,16 & 4,98 & 4,95 \\
\hline Nivel de creatividad & 3,92 & 4,78 & 4,52 & 5,07 & 4,75 & 4,61 \\
\hline $\begin{array}{l}\text { Nivel de resolución de } \\
\text { problemas }\end{array}$ & 4,42 & 4,72 & 4,89 & 4,84 & 5,10 & 4,79 \\
\hline $\begin{array}{l}\text { Nivel de liderazgo } \\
\text { y capacidad de } \\
\text { comunicación }\end{array}$ & 3,33 & 4,26 & 3,97 & 4,48 & 3,60 & 3,93 \\
\hline $\begin{array}{l}\text { Nivel de desarrollo de } \\
\text { nuevos productos y } \\
\text { servicios }\end{array}$ & 4,42 & 4,22 & 3,40 & 4,09 & 3,88 & 4,00 \\
\hline $\begin{array}{l}\text { Nivel de estableci- } \\
\text { miento de relaciones y } \\
\text { contactos profesionales }\end{array}$ & 4,42 & 4,53 & 4,51 & 4,57 & 4,38 & 4,48 \\
\hline $\begin{array}{l}\text { ¿Ha considerado } \\
\text { alguna vez } \\
\text { seriamente convertirse } \\
\text { en empresario? }\end{array}$ & 1,17 & 1,27 & 1,35 & 1,45 & 1,37 & 1,32 \\
\hline
\end{tabular}

En la Tabla 11, se visualiza que la experiencia laboral (desde 1 desacuerdo total a 7, acuerdo total) es mínima. Así mismo, las empresas donde han trabajado no pasan el promedio de cien trabajadores. El autoempleo (1- si, 2- no, su media seria 1.5) no es muy atractivo para los alumnos. Y en el caso de tenerlo, la mayoría ha permanecido cerca de un año en el mismo. La formación técnica y de emprendimiento presentan valores atractivos, los encuestados presentan mayor conocimiento en el sector público. 
TABLA 11. FORMACIÓN, EXPERIENCIA Y CONOCIMIENTO EMPRESARIAL A PARTIR DE LA ENCUESTA REALIZADA A ALUMNOS MATRICULADOS EN EL SEMESTRE B-2014 DE LA UCSG

\begin{tabular}{|c|c|c|c|c|c|c|}
\hline CARRERA & 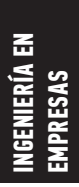 & 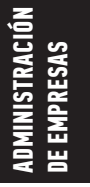 & 중 & 을 & 豆兽 & 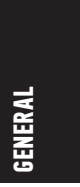 \\
\hline $\begin{array}{l}\text { ¿Tiene alguna } \\
\text { experiencia laboral? }\end{array}$ & 2,43 & 2,16 & 1,65 & 4,13 & 2,54 & 2,58 \\
\hline $\begin{array}{l}\text { ¿Cuánto tiempo de } \\
\text { experiencia laboral } \\
\text { tiene? (años) }\end{array}$ & 1,15 & 1,24 & 1,11 & 2,32 & 3,10 & 1,79 \\
\hline
\end{tabular}

Trabajadores/

$\begin{array}{llllll}\text { trabajadoras que tiene } \quad 84,4 & 65,87 & 45,23 & 36,5 & 89,7 & 64,34\end{array}$

donde trabajo

\begin{tabular}{|c|c|c|c|c|c|c|}
\hline $\begin{array}{l}\text { ¿Ha estado alguna vez } \\
\text { auto empleado? }\end{array}$ & 1,84 & 1,67 & 1,58 & 1,45 & 1,81 & 1,67 \\
\hline $\begin{array}{l}\text { ¿Cuánto tiempo ha } \\
\text { estado auto empleado? } \\
\text { (años) }\end{array}$ & 1,08 & 1,42 & 1,12 & 1,67 & 1,85 & 1,43 \\
\hline
\end{tabular}

$\begin{array}{lllllll}\begin{array}{l}\text { Grado de conocimiento } \\ \text { sobre organización } \\ \text { privadas }\end{array} & 3,08 & 4,24 & 3,45 & 3,84 & 4,05 & 3,73 \\ \end{array}$

Grado de conocimien-

tos sobre organismos $\quad 4,83 \quad 4,53 \quad 4,55 \quad 4,55 \quad 4,35 \quad 4,56$

públicos

\begin{tabular}{|c|c|c|c|c|c|c|}
\hline $\begin{array}{l}\text { Grado de conocimiento, } \\
\text { medidas de formación } \\
\text { específica para } \\
\text { jóvenes empresarios }\end{array}$ & 5,33 & 5,63 & 5,42 & 5,8 & 5,45 & 5,52 \\
\hline $\begin{array}{l}\text { Grado de conoci- } \\
\text { miento, prestamos en } \\
\text { condiciones especial- } \\
\text { mente favorables }\end{array}$ & 2,67 & 2,84 & 2,94 & 3,45 & 3,19 & 3,02 \\
\hline $\begin{array}{l}\text { Grado de conocimien- } \\
\text { to, ayuda técnica para } \\
\text { iniciar negocio }\end{array}$ & 5,50 & 5,12 & 5,20 & 4,75 & 4,74 & 5,06 \\
\hline $\begin{array}{l}\text { Grado de conocimiento, } \\
\text { creación de empresas }\end{array}$ & 4,08 & 4,65 & 4,14 & 4,93 & 4,40 & 4,44 \\
\hline
\end{tabular}

CONCLUSIONES Y RECOMENDACIONES

La actitud empresarial de los alumnos, que en mayoría son de cursos básicos, es aceptable. La carrera de Administración de Empresas (la cual concentra mayor cantidad de alumnos) presenta mayor predisposición hacia el emprendimiento. La influencia de la sociedad, específicamente de sus compañeros de estudio, resulta muy importante al momento de ser emprendedor. La valoración social, mayormente de desaprobación del emprendimiento empresarial, resulta irrelevante puesto que la familia, compañeros y amigos son la mayor influencia para los encuestados.

La CEA proyecta estudiantes con conocimiento en emprendimiento. Las madres de los encuestados representan el modelo emprendedor en el hogar. También es importante el ámbito, de tal forma que las oportunidades de éxito generan el aumento de la actitud emprendedora por encima de la familia, amigos y compañeros. En sí, el alumno tiene predisposición interna y externa hacia el emprendimiento.

En lo social, la percepción de la ciudadanía no es importante para el alumnado y además se presentan datos favorables acerca del grado de conocimiento en actividades empresariales, siendo el sector público el más codiciado. Debido a que la muestra en su mayoría, presenta características de un público nuevo en el sistema universitario, es de entender la poca experiencia que reflejan los datos. Los estudiantes que trabajan, en su mayoría, lo hacen en empresas formadas por otros individuos.

La influencia que la universidad en su conjunto tiene sobre el alumno se traduce en varios ámbitos, como el educativo, administrativo y el entorno estudiantil. Este último muy importante para el crecimiento personal de cada uno, ya que los alumnos generan contactos para futuro, explícita e implícitamente, de tal forma que la red de contactos nace o crece en las aulas.

El presente estudio busca generar réplicas en otras unidades educativas, sin discriminación de tipo, para su comparación y a la vez, para obtener resultados generales que permitan conocer el grado de influencia que cada aspecto estudiado tiene sobre el alumno. Ya que somos un país diverso, sería interesante poder comparar datos no solo entre las facultades de la UCSC, sino también en universidades de otras provincias o regiones del Ecuador.

\section{REFERENCIAS BIBLIOGRÁFICAS}

Ajzen, I. (1991). The theory of planned behaviour. Organizational Behaviour and Human Decsions Processes, 50(1), 179-211.

Amit, R. (1997). Charla de intraempresario. Sydney: Conferencia Internacional de Emprendimiento.

Bennis, W., \& Nanus, B. (1985). Leaders: the strategies for taking charge. New York: Harper and Row Publisher.

Cruz, N. M., Barahona, J. J., \& Escudero, A. I. (2005). Análisis de la formación y la experiencia laboral como determinantes del espíritu emprendedor de los estudiantes universitarios. RAE: Revista Asturiana de Economía, 34, 131-145.

Delmar, F., \& Davidsson, P. (2000). Where do they come from? Prevalence and characteristics of nascent entrepeneurs. Entrepeneurship and Regional Development, 12(1), $1-23$. 
Galicia, F. A. (1990). Introduccion a la metodología de investigacion en ciencias de la administración y del comportamiento: contabilidad, economía, administración, psicología, sociología, trabajosocial. México D.F.: Trillas.

Hernández, R., Fernández, C., \& Baptista, M. d. (2010). Metología de la Investigación (15 ed.). México D.F.: Mc Graw Hill.

Kantis, H., Angelelli, P., \& Moori, V. (2004). Desarrollo emprendedor: América Latina y la experiencia internacional. New York: IDB.

Karimi, S., Chizari, M., Biemans, H., \& Mulder, M. (2010). Entrepreneurship Education in Iranian Higher Education: The Current State and Challenges. European Journal of Scientific Research, 48(1), 35-50.

Laso, V., Caicedo, G., Ordeñana, X., \& Izquierdo, E. (2015). Clobal Entrepreneurship Monitor Ecuador 2014. Guayaquil: ESPAE.

Lind, D. M. (2012). Statistical Techniques in Business \& Economics. New York: McGraw-Hill/Irwin.

Liñán, F., Urbano, D., \& Guerrero , M. (2011). Regional variations in entrepreurial cognitions: Start-up intentions of universitystudentsinSpain. Entrepeneurship \& Regional Development, 23, 187-215.

Méndez, O. J., Miramontes, B. L., \& Monreal, Z. A. (2012). Intención de emprendimiento en estudiantes: el caso de una universidad pública en ciudad Juárez, México. Global Conference on Business \& Finance Proceedings, 7, págs. 1231-1243.

Mintzberg, H., Ahlstrand, B., \& Lampel, S. (1999). Safarialaestrategia. Buenos Aires: Ediciones Gránica.

Mitchell, R. K., Busenitz, L., Lant, T., Mcdougall, P. P., Morse, E. A., \& Smith, J. B. (2002). Toward a theory of entrepeneurial cognition: Reythinking the people side of entrepeneurship research research. Entrepeneurship Theory and Practice 27(2), 93-104.

Montiel, O., Márquez, B., Arámbula, Z., \& Ordóñez, L. (2012). Intenciondeemprendimientoen estudiantes: El caso de una universidad pública de Ciudad Juárez, México. Global Conference, 7(1), 1231-1244.
Olmos, R. E., \& Castillo, M. Á. (2007). La actitud emprendedora durante la vida académica de los estudiantes universitarios. Cuadernos de estudios empresariales, 17, 95-116.

Reynolds, P., Hayand, M., \& Camp, S. (1999). Global Entrepreneurship Monitor, 1999 Executive Report. Kansas City: Center for Entrepreneurial Leadership at the Ewing Marion Kauffman Foundation.

Rodríguez, A. (2009). Nuevas perspectivas para entender el emprendimiento empresarial. Pensamiento y Gestión (26), 94-119.

Samaja, J. (2007). Epistemología y metodología: elementos para una teoría de la investigación científica. Buenos Aires: Eudeba.

Shapero, A., \& Sokol, L. (1982). Social dimensions of entrepeneurship. En C. A. Kent, D. L. Sexton, \& K. H. Vesper, Encyclopedia of 835 entrepeneurship (págs. 72-90). Englewood Cliffs, New Jersey: Prentice-Hall.

Singer, S., Amorós, J. E., \& Arreola, D. M. (2015). Global entrepreneurship monitor: 2014 global report. Global Entrepreneurship Research Association. Zabreb: GEM.

Smit, A. B. (2004). Changing external conditions requirehigh levels of entepreneurship in agriculture. Berlin: International Society for Horticultural Science Publication.

Vérin, H. (1982). Entrepreneurs, entreprise, histoire d’une idée. Avant-propos In, 2, 9-14.

Wallace, I., \& Nilsson, E. (1997). The role of agricultural education and training in improving the performance of support services for the renewable natural resources sector (Vol. 24). Reino Unido: Overseas Development Institute.

Yin, R. K. (2003). Case study research, Design and Methods (Tercera ed.). Los Angeles: Sage Publications.

Zoltan, A. C. (2006). How isentrepreneurshipgood for economic growth? Innovations: Technology, Governance, Globalization, 1, 97-107. 